\title{
Ultrasonography Appearances of Hepatic Metastases from Female Breast Cancer in Patients in Central Sudan
}

\author{
Suliman Salih, ${ }^{1,2}$ Ahmed Elhaj ${ }^{2}$ \\ ${ }^{1}$ Faculty of Applied Medical Sciences, Taibah University, Saudi Arabia \\ ${ }^{2}$ National Cancer Institute - University of Gezira, Sudan
}

\begin{abstract}
:
Background: Breast cancer is the most prevalent cause of cancer-related death both worldwide and in Sudan. Metastases from breast cancer most frequently occur in the liver.

Objective: The purpose of this study was to evaluate the appearance of liver metastases on ultrasonography images in female breast cancer patients in Central Sudan.

Materials and methods: Between 2002 and 2007, 108 breast cancer patients diagnosed with liver metastases underwent grey scale ultrasonography. Data were analyzed using Microsoft Excel 2010, software.

Results: Of these 108 patients, multiple liver metastases were observed on ultrasonography in $92 \%$ of cases, whereas a single metastasis was observed in the remaining $8 \%$ cases. Liver metastases from breast cancer showed hypoechoic, hyperechoic, mixed (hypoechoic and hyperechoic), and isoechoic signals in $70 \%, 21 \%$, $6 \%$, and $3 \%$ of cases, respectively.
\end{abstract}

Conclusion: Ultrasonography improves the detection and characterization of multiple metastases with a hypoechoic pattern.

Keywords: Breast cancer, liver metastases, echogenicity patterns, ultrasonography

\section{Introduction:}

Breast cancer is the most commonly diagnosed malignancy and the leading cause of cancer death in women worldwide. More than half of all breast cancers are diagnosed in developing countries, where its incidence is increasing due to changes in reproductive factors and lifestyle and increased life expectancy [1,2]. It has also been found that, in developing countries, breast cancer is diagnosed at later stages, and more frequently occurs in young and premenopausal women who reside in rural areas[3-5]. In addition, there is limited access to cancer treatment, including breast cancer therapy, in these regions. This is a result of insufficient numbers of trained health care professionals, the limited availability and high cost of cancer drugs, the inadequacy of existing equipment, and a lack of modern equipment including radiotherapy machines [6,7].

Cancer metastasis is the main cause of cancer mortality. The liver is the most frequent site of metastasis via the hematogenous route and is involved in up to one-third of cases of metastasizing cancers. The incidence and prevalence of liver metastases from breast cancer has previously been reported to range from $1.5 \%$ to $20 \%$. Liver metastases are associated with a poor clinical outcome [8-10].

A number of factors have previously been shown to affect the pattern metastatic breast cancer spread including estrogen receptor status, tumor histological type, and the use of adjuvant chemotherapy. Multiple studies have shown that the tumor histological grade is an important prognostic marker both in primary breast cancer and in women who develop metastatic disease [11-13].

Despite significant advances its detection and treatment, breast cancer continues to have a significant impact on health in the United States, with an estimated 234,580 individuals newly diagnosed with this disease in 2013 [14]. Approximately 33\% of individuals with breast cancer diagnosed between 2001 and 2007 had regional metastases, with a 5-year relative survival rate of $84 \%$. Approximately $5 \%$ were diagnosed with distant metastases, most commonly in the bones, lungs, liver, or brain, and had a 5-year relative survival rate of only 23\%[14]. In developing countries, women diagnosed with breast cancer are on average younger than those in developed countries), and have later stage disease (III or IV, i.e. with axillary node involvement and distant metastases) [15].

Several imaging modalities, including ultrasonography, magnetic resonance imaging (MRI), computed tomography (CT), positron emission tomography (PET), PET-CT, and bone scintigraphy, are used to diagnose metastases and evaluate the effects of treatment for metastatic breast cancer [8]. However, as outlined in the guidelines by the National Comprehensive Cancer Network and the National Institute for Health and Clinical Excellence, evidence of the accuracy and effectiveness of these modalities in evaluating the response to treatment of metastatic breast cancer is lacking, even though the type and finding of imaging studies may strongly affect patient outcomes [16]. Furthermore, the inappropriate use of these imaging modalities could lead to overtreatment. For example, the use of MRI during breast cancer diagnosis and treatment planning is 
associated with longer time intervals from diagnosis to treatment [17, 18] and with potentially avoidable mastectomies [19, 20]. However, the inappropriate use of imaging may lead to under treatment if additional disease foci are not identified and lead to disease progression. Furthermore, imaging modalities vary substantially in cost, with very high direct costs for MRI but far lower direct costs for ultrasonography, and availability and cost effectiveness may determine the imaging modality that is used [21, 22]. Ultrasonography is the most cost-effective imaging modality and is widely used in developing countries due to budget constraints and the unavailability of advanced CT and MRI machines [23].

Ultrasonography of the liver results in hypoechoic, hyperechoic, and mixed signals [24]. The most common hypoechoic liver metastases are from the breast, lung, esophagus, stomach, pancreas, and non-Hodgkin lymphoma. Multiple lesions are present in approximately $98 \%$ of patients, and both lobes are often involved. These metastases are usually hypovascular on Doppler evaluation [25].

The purpose of this study was to assess the patterns and echogenicity of liver metastases on ultrasonography amongst female breast cancer patients in central Sudan.

\section{Methods:}

A series of 108 female patients with breast cancer and liver metastases was retrospectively reviewed. The mean age of patients was 44 years (range, 27-81 years). The study was conducted at the National Cancer Institute, at Gezira University, Central Sudan using grey scale ultrasonography data retrieved from the registry of cancer patients diagnosed between 2002 and 2007. The review included the liver ultrasonography patterns (single or multiple) and echogenicity patterns (hypoechoic, hyperechoic or mixed), according to radiologists` reports in the patients file (Fig. 1). Data were analyzed using Microsoft Excel 2010, software.

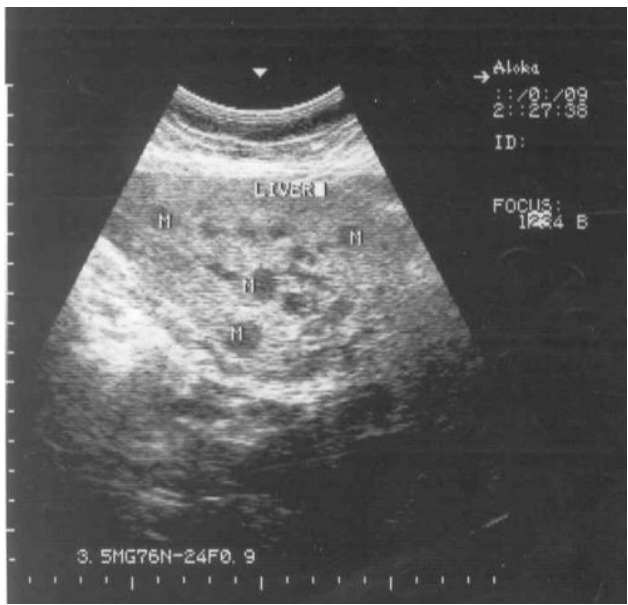

Figure 1. An example of liver metastases on an ultrasonography image. Liver ultrasonography of a 47-year-old woman with cancer of the left breast showed multiple metastases with hypoechoic echogenicity.

\section{Results:}

Multiple liver metastases were apparent upon ultrasonography in $92 \%$ of cases, and a single metastasis was present in $8 \%$ of cases (Fig 2). The echogenicity of the liver metastases from breast cancer was hypoechoic, hyperechoic, mixed (hypoechoic and hyperechoic), and isoechoic in 70\%, 21\%, 6\%, and 3\% of cases, respectively (Fig 3).

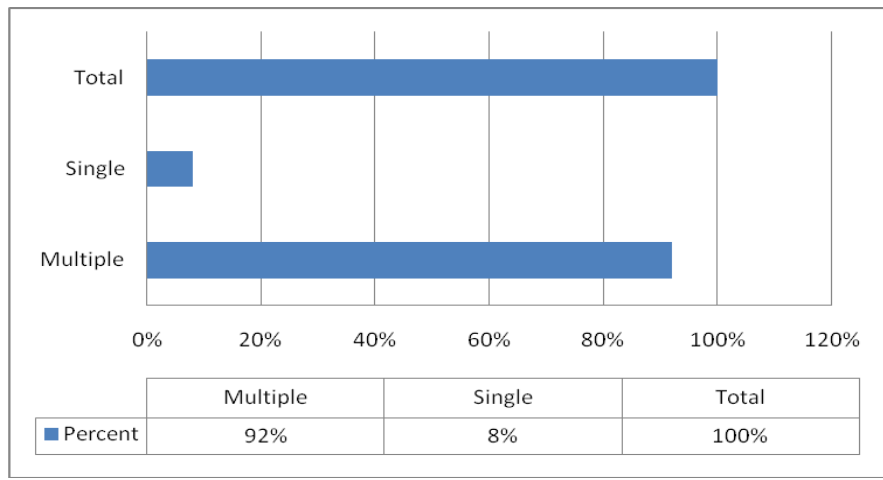

Figure 2. Multiplicity of liver metastases 


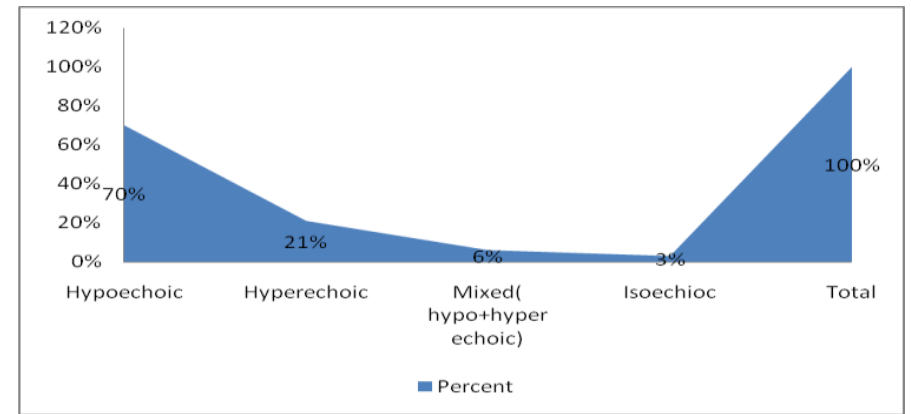

Figure 3. Echogenicity of breast cancer liver metastases.

\section{Discussion:}

Brightness (B)-mode ultrasonography is highly sensitive, specific, and cost effective in the diagnosis and follow up of patients with liver metastases, particularly in developing countries. The high cost of more advanced imaging modalities such as MRI and CT limits their availability in these regions.

The result revealed that $92 \%$ of the liver metastases patterns are multiple, these findings are supported by those of $\mathrm{Li}$ and Hann, who found that $98 \%$ of breast cancer metastases are multiple features and involved both lobes [25], and close correlated with study conducted by Yoshida T et a. [26]. Our study showed that breast cancer liver metastases could have a variety of ultrasonography echogenic appearances; hypoechoic (70\% of cases), hyperechoic (21.7\% of cases), mixed (hypo and hyperechoic; 5.7\% of cases), and isoechoic (3.8\% of cases). These findings are in accordance with a study by Bruneton et al. who found that about $70 \%$ of liver metastases had a hypoechoic appearance on ultrasonography [27]. The most common hypoechoic liver metastases were from breast, lung, esophagus, stomach, and pancreatic cancer and non-Hodgkin lymphoma. These metastases were usually hypovascular on Doppler evaluation [28].

The high prevalence of hypoechoic findings will influence the choice of low cost imaging studies, such as ultrasonography, which is comparable with other advanced imaging methods including MRI and CT, particularly when used with contrast media. This opinion is shared by Westwood et al. and Guang et al who reported that contrast - enhanced ultrasound (CEUS) could provide a similar diagnostic performance to other imaging modalities contrast- enhanced computed tomography (ECT) and contrast- enhanced magnetic resonance imaging (CEMRI) for the assessment of focal liver lesions (FLLs) [29,30]. Ultrasonography can play important role in staging and follow-up of breast cancer patients, particularly in developing countries, where resources for advanced medical imaging is limited [31].

\section{Conclusion:}

In this study, we found that $70 \%$ of breast cancer metastases in the liver had a hypoechoic appearance, whilst the remaining $21 \%, 6 \%$, and $3 \%$ of metastases had a hyperechoic, mixed, and isoechoic appearance, respectively. Ultrasonography is a cheap, safe and effective method of detecting focal liver lesions.

\section{Declaration of interest:}

The authors declare that they have no conflict of interest.

\section{Acknowledgement:}

We would like to thank all staff of cancer registry unit at National cancer Institute, Gezira University, Sudan, for their assisting in the data collection of this manuscript. Our thank also extended to Dr. Mohamed Yousef for revising the manuscript.

\section{References}

[1]. Beaulieu N, Bloom D, Bloom R, Stein R. Breakaway: The Global Burden of Cancer - Challenges and Opportunities. London, UK: The Economist Intelligence Unit; 2009.

[2]. Ngoma TA. World Health Organization cancer priorities in developing countries. Ann Oncol. 2006;17:viii9-viii14.

[3]. Porter P. "Westernizing” women's risks? Breast cancer in lower-income countries. N Engl J Med. 2008;358:213-216.

[4]. Ly M, Antoine M, André F, Callard P, Bernaudin JF, Diallo DA. Breast cancer in Sub-Saharan African women: review. Bull Cancer 2011;98:797-806.

[5]. Elgaili EM, Abuidris DO, Rahman M, Michalek AM, Mohammed SI. Breast cancer burden in central Sudan. Int J Womens Health. 2010;2:77-82. 
[6]. Ferlay J, Shin HR, Bray F, et al. Estimates of worldwide burden of cancer in 2008: GLOBOCAN 2008. Int J Cancer. 2010;127:2893-2917.

[7]. Ferlay J, Shin HR, Bray F, et al. GLOBOCAN 2008 v1.2, Cancer Incidence and Mortality Worldwide: IARC CancerBase No. 10 [Internet]. Lyon, France: International Agency for Research on Cancer, 2010. Available from: http://globocan.iarc.fr. Accessed January, 2014. WHO Global Health Observatory. 2010, World Health Organisation.

[8]. Myers RE, Johnston M, Pritchard K et al. Baseline staging tests in primary breast cancer: a practice guideline. Can Med Assoc J 2001;164:1439-1444.

[9]. Gerber B, Seitz E, Muller H, et al. Perioperative screening for metastatic disease is not indicated in patients with primary breast cancer and no clinical signs of tumor spread. Breast Cancer Res Treat 2003;82:29-37.

[10]. Wells PNT. Ultrasonics in Clinical Diagnosis. 2nd edn. Churchill Livingstone, London. [London Bisset, Khan and Sabih. Differential Diagnosis in Abdominal Ultrasound, 3/edition (2008). Elsevier.

[11]. Elston CW, Ellis IO. Prognostic factors in breast cancer. The value of histological grade in breast cancer: experience from a large study with long-term follow-up. Histopathology. 1991;19:403-410.

[12]. Robertson JFR, Dixon AR, Nicholson RI, et al. Confirmation of a prognostic index for patients with metastatic breast cancer treated by endocrine therapy. Breast Cancer Res Treat 1992;22:221-227.

[13]. Blanco G, Hollf K, Heikkin M, et al. Prognostic factors in recurrent breast cancer: relationships to site of recurrence, disease free interval, female sex steroid receptors, ploidy and histological malignant grading. Br J Cancer 1990;62:142-146.

[14]. American Cancer Society. Cancer Facts \& Figures 2013. Atlanta, GA: 2013.

[15]. Ly M, Antoine M, André F, Callard P, Bernaudin JF, Diallo DA. Breast cancer in Sub-Saharan African women: review. Bull Cancer. 2011;98:797-806.

[16]. National Institute for Health and Clinical Excellence (NICE). Advanced Breast Cancer: Diagnosis and Treatment National Collaborating Centre for Cancer National Institute for Health and Clinical Excellence. London: 2009. http://www.nice.org.uk/nicemedia/live/11778/43305/43305.pdf. Accessed January 2014.

[17]. Bleicher R, Ciocca R, Egleston B, et al. Association of routine pretreatment magnetic resonance imaging with time to surgery, mastectomy rate, and margin status. J Am Coll Surg 2009;209:180-187.

[18]. Hulvat M, Sandalow N, Rademaker A, et al. Time from diagnosis to definitive operative treatment of operable breast cancer in the era of multimodal imaging. Surgery 2010;148:746-750; discussion 750-751.

[19]. Pettit K, Swatske M, Gao F, et al. The impact of breast MRI on surgical decision-making: are patients at risk for mastectomy? J Surg Oncol 2009;100:553-558.

[20]. Houssami N, Ciatto S, Macaskill P, et al. Accuracy and surgical impact of magnetic resonance imaging in breast cancer staging: systematic review and meta-analysis in detection of multifocal and multicentric cancer. J Clin Oncol 2008;26:3248-3258

[21]. Centers for Medicare and Medicaid Services (CMS). Physician Fee Schedule. 2009. http://chfs.ky.gov/nr/rdonlyres/4fd6f67c-6c974061-a9fd-2441467201f2/0/mdfee2009rev4909r2.pdf. Accessed October 9, 2013.

[22]. Centers for Medicare and Medicaid Services (CMS). Medicare Program: Changes to the Hospital Outpatient Prospective Payment System and CY 2009 Payment Rates CMS. 2009. http://www.cms.gov/Medicare/Medicare-Fee-for-ServicePayment/HospitalOutpatientPPS/downloads/CMS-1404-FC.pdf. Accessed April 9, 2014.

[23]. Ferlay J, Shin HR, Bray F, et al. Estimates of worldwide burden of cancer in 2008: GLOBOCAN 2008. Int J Cancer 2010; 127:2893-2917.

[24]. Wells PNT. Ultrasonic in Clinical Diagnosis. $2^{\text {nd }}$ edition Churchill Livingstone, London] [London Bisset, Khan and Sabih. Differential Diagnosis in Abdominal Ultrasound, 3/edition (2008) Elsevier.

[25]. Li D and Hann L. A Practical Approach to Analyzing Focal Lesions in the Liver, Ultrasound Q 2005; 21:187-200.

[26]. Yoshida T, Matsue H, Okazaki N, Yoshino M. Ultrasonographic differentiation of heapatocellular carcinoma from metastatic liver cancer. J Clin Ultrasound.1987; 15(7):431 - 437.

[27]. Bruneton JN, Ladree D, Caramella E, et al: Ultrasonographic study of calcified hepatic metastases: a report of 13 cases. Gastrointest Radiol. 1982; 7:61-63.

[28]. Wernecke K, Vassallo P, Bick U et-al. The distinction between benign and malignant liver tumors on sonography: value of a hypoechoic halo. AJR Am J Roentgenol. 1992; 159 (5): 1005-9.

[29]. Westwood M, Joore M, Grutters J, Redekop K, Armstrong N, Lee K, Gloy V, Raatz H, Misso K, Severens J, Kleijnen J.Contrastenhanced ultrasound using SonoVue ${ }^{\circledR}$ (sulphur hexafluoride microbubbles) compared with contrast-enhanced computed tomography and contrast-enhanced magnetic resonance imaging for the characterisation of focal liver lesions and detection of liver metastases: a systematic review and cost-effectiveness analysis. Health Technol Assess. 2013 Apr;17(16):1-243.

[30]. Guang Y, Xie L, Ding H, Cai A, Huang Y . Diagnosis value of focal liver lesions with SonoVue ${ }^{\circledR}$-enhanced ultrasound compared with contrast-enhanced computed tomography and contrast-enhanced MRI: a meta-analysis. J Cancer Res Clin Oncol. 2011 Nov; 137(11):1595-60.

[31]. Yang WT. Staging of breast cancer with ultrasound. Semin Ultrasound CT MR. 2011;32(4):331-41. 